\title{
MicroRNA-26a inhibits proliferation and metastasis of human hepatocellular carcinoma by regulating DNMT3B-MEG3 axis
}

\author{
YARUI LI* ${ }^{*}$ MUDAN REN*, YAN ZHAO, XINLAN LU, MENGYAO WANG, \\ JUNBI HU, GUIFANG LU and SHUIXIANG HE
}

Department of Gastroenterology, The First Affiliated Hospital of Xi'an Jiaotong University, Xi'an, Shaanxi 710061, P.R. China

Received December 7, 2016; Accepted April 5, 2017

DOI: 10.3892/or.2017.5579

\begin{abstract}
R-26a is known to play an important oncosuppressive role in HCC. However, its regulatory role and relationship with other non-coding RNAs is less clear. In the present study, we report that the expression levels of miR-26a and long non-coding RNA (IncRNA) maternally expressed gene 3 (MEG3) were frequently downregulated in HCC tissues compared to matched non-malignant tissues. In addition, the expression levels of miR-26a and MEG3 were negatively correlated with the tumor sizes and TNM clinical stage in HCC patients. Overexpression of miR-26a significantly reduced the capacity of proliferation, invasion and migration of HCC cells. Moreover, we demonstrated that DNA methyltransferase 3b (DNMT3B) was a direct target gene of miR-26a. Overexpression of miR-26a suppressed the expression level of DNMT3B. Inhibited expression of DNMT3B showed similar tumor suppressive effects induced by miR-26a upregulation, and resulted in the upregulation of MEG3. Furthermore, we found that the expression levels of DNMT3B were upregulated in the HCC tissues compared with non-malignant tissues, and it was inversely correlated with miR-26a and MEG3 in HCC tissues. Thus, these results provided a plausible link between the observed reduction of miR-26a and MEG3 in HCCs. Together, the present study added miR-26a/DNMT3B/MEG3 axis to the complex mechanisms of HCC development.
\end{abstract}

\section{Introduction}

Hepatocellular carcinoma (HCC) is the sixth most frequent diagnosed cancer and one of the most common cause of

Correspondence to: Dr Shuixiang He or Guifang Lu, Department of Gastroenterology, The First Affiliated Hospital of Xi'an Jiaotong University, Xi'an, Shaanxi 710061, P.R. China

E-mail: hesx123@126.com

E-mail: lugf929@126.com

*Contributed equally

Key words: miR-26a, DNMT3B, MEG3, hepatocellular carcinoma cancer-related death worldwide $(1,2)$. Although the diagnostic and therapeutic techniques of HCC have gradually improved, the morbidity and mortality of HCC continue to show an upward trend (3-6). The potential molecular mechanisms are critical for understanding the development and progression of HCC (7). Therefore, we need to further understand the pathogenesis of HCC and identify novel molecular markers to improve the diagnosis and treatment of HCC.

Among the large scale of mammalian genome, only a small fraction of transcripts represent protein-coding genes (8), the involvement of non-protein coding genes also act as critical regulators in pathogenesis and growth of various human diseases including cancer processes $(9,10)$. MicroRNAs (miRNAs), a group of short non-coding RNAs (20-25 nucleotides) that have been extensively studied and identified to be crucial transcriptional regulators of carcinogenesis $(11,12)$. miRNAs regulated target gene expression by induce messenger RNAs (mRNA) degradation and inhibit the translation and stability of mRNAs through binding to the 3'-untranslated region (3'-UTR) $(13,14)$. Numerous miRNAs were identified from thousands of cancer related studies serving as promising biomarkers or therapeutic targets $(15,16)$. For instance, miR-21, miR-124, miR-148a and miR-384 have been reported to be involved in the progression of HCC (17-20). In recent years, emerging studies showed that aberrant expression of long noncoding RNAs (lncRNAs), a class of ncRNAs more than 200 nucleotides in length, was involved in the tumorigenesis and development of various cancer, including HCC (21-24). Present research demonstrated that IncRNA function as competing endogenous RNAs (ceRNAs) or act as molecular 'sponges' to regulate the concentration, expression and biological functions of miRNAs (25). For example, Hu et al (26) found that long non-coding RNA GAS5 suppressed the migration and invasion of HCC cells through negative regulation of miR-21 and its downstream targets. Nevertheless, the underlying mechanism for the miRNA/lncRNA trans-regulatory function in $\mathrm{HCC}$ remains unclear.

miR-26a has been reported to act as tumor suppressor via targeting specific downstream genes in several human cancers, such as pancreatic, breast, bladder and gastric cancer (27-30). MEG3, one of the most significantly downregulated lncRNAs in HCC, encodes a long non-coding RNA of 1700 nucleotides and function as a tumor-suppressor in HCC (31). Notably, the study also demonstrated that miR-29a could regulate the 
expression level of MEG3 through the modulation of DNMT3B activity in HCC (31). In the present study, we identified that both miR-26a and MEG3 were significantly decreased in HCC compared to matched non-malignant tissues and showed negative correlation between them. This leads to the hypothesis that miR-26a could also silence the expression of MEG3 in HCC via similar mechanism. Therefore, we conducted the present study to investigate the role of miR-26a and examined whether miR-26a suppresses HCC growth and metastasis by targeting DNMT3B/MEG3 axis.

\section{Materials and methods}

Samples and cell line growth conditions. Forty-six patients who received curative resection for $\mathrm{HCC}$ at the First Affiliated Hospital of Xi'an Jiaotong University between June 2015 and May 2016 were enrolled in the study. None of the patients received any chemotherapy or radiotherapy treatments before surgery. Tumor tissues and matched adjacent non-malignant tissues (3-5 cm distal to the edge of tumor) were immediately frozen in liquid nitrogen or stored at $-80^{\circ} \mathrm{C}$ until RNA extraction. All patients signed the informed consent and the study was approved by the Institutional Ethics Committee. The human HCC cell lines (HepG2, Hep3B, MHCC97-H and SMCC-7721) and normal liver cell line (LO-2) were obtained from the Shanghai Institutes of Biological Sciences (Shanghai, China). Cells were grown in Dulbecco's modified Eagle's medium (DMEM; Gibco-Invitrogen, Carlsbad, CA, USA) supplemented with $10 \%$ fetal bovine serum (FBS) and $1 \%$ antibiotics, cultured at $37^{\circ} \mathrm{C}$ in $5 \% \mathrm{CO}_{2}$ humidified incubator.

RNA isolation and quantitative real-time polymerase chain reaction ( $q R T-P C R)$. Total RNA was isolated from specimens and cultured cells by using TRIzol reagent (Invitrogen) according to the manufacturer's protocol. qRT-PCR were used to detect the expression levels of miRNA, mRNA and lncRNA on Thermal Cycler Dice Real-Time system. The required reagents, including Mir-X miRNA qRT-PCR SYBR kit, PrimeScript RT Master Mix and SYBR Premix Ex Taq ${ }^{\mathrm{TM}}$ II, were purchased from Takara Bio (Shiga, Japan). The primers for specific genes were as follows: DNMT3B-F, 5'-GCTCTTA CCTTACCATCG-3'; DNMT3B-R, 5'-GATACTCTGAACT GTCTCC-3'. MEG3-F, 5'-GGCTGAAGAACTGCGGATG-3'; MEG3-R, 5'-CCAGGAAGGAGACGAGAGG-3'. Bio-Rad CFX Manager 2.1 software was used for analysis of qRT-PCR. All experiments were performed in triplicate and $2^{-\Delta \Delta \mathrm{Ct}}$ was regarded as the analytic formula.

Cell transfection. hsa-miR-26a mimics and negative control (NC) were purchased from Guangzhou RiboBio, Co., Ltd. (Guangzhou, China). Small interfering RNA against DNMT3B and negative control (NC) were designed by Shanghai GenePharma, Co., Ltd. (Shanghai China). The sequences of the si-DNMT3B were as follows: sense 5'-GUACCAUGCUCUGGAGAAATT-3' and antisense 5'-UUUCUCCAGAGCAUGGUA CTT-3'. Both HepG2 and MHCC97-H cells were seeded onto 6-well plates, ensured $80 \%$ confluence at the time of transfection. Transfection was carried out using Lipofectamine 2000 (Invitrogen) method following the manufacturer's protocol.
Cell viability assay. MTT (3-(4,5-dimethylthiazol-2-yl)-2, 5-diphenyltetrazolium bromide) assay was used to measure cell proliferation. In Brief, HepG2 and MHCC97-H cells were seeded in 96-well plates at a density of 6,000 cells/well, incubated in appropriate medium. After transfection for 24, 48, 72 and $96 \mathrm{~h}$, cells were subsequently cultured in medium with $0.5 \mathrm{mg} / \mathrm{ml}$ MTT for $4 \mathrm{~h}$. Following removal of the supernatant and $150 \mu 1$ dimethyl sulfoxide (DMSO) was added. Then the optical density (OD) at $490 \mathrm{~nm}$ was determined by EnSpire Multimode plate reader (Perkin-Elmer, Waltham, MA, USA).

Transwell assay. Transwell assay was performed to assess cell migration and invasion after transfection for $48 \mathrm{~h}$. For invasion, the upper chambers were pre-coated with Matrigel

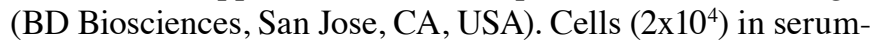
free medium were seeded into the top chambers of an insert ( $8 \mu \mathrm{m}$ pore size; Merck Millipore, Billerica, MA, USA), which were soaked into the bottom chambers filled with complete medium. For migration, Matrigel was not needed to coat the upper membrane, then the same invasion assay was performed. After $48 \mathrm{~h}$ of incubation, the chambers were fixed by $4 \%$ paraformaldehyde and then stained with $0.1 \%$ crystal violet. The cells passing through the film were observed under an optical microscope, 10 field-of-view counts were selected and the mean was calculated.

Western blot analysis. Proteins were collected from cells using RIPA buffer (Pierce, Rockford, IL, USA), then separated by $12 \%$ SDS-PAGE and transferred to the PVDF membrane (Merck Millipore). The membrane was blocked with 5\% non-fat milk and incubated with primary antibody DNMT3B (Abcam, Cambridge, MA, USA) and $\beta$-actin (Santa Cruz Biotechnology, Santa Cruz, CA, USA) with 1:1,000 dilution at $4^{\circ} \mathrm{C}$ overnight. Then the membrane was washed extensively followed by incubation with secondary antibodies (Zhuangzhi Biotech, Co., Ltd., Xi'an, China) at room temperature for $1 \mathrm{~h}$. The results were obtained with chemiluminescent (Pierce). ImageJ software-based analysis was used to quantify the bands obtained through western blot analysis.

Luciferase reporter assay. Cells were cultured in 96-well plates with $50-70 \%$ confluence $24 \mathrm{~h}$ before transfection. A mixture of $120 \mathrm{ng}$ plasmid vector including the wild-type (WT) or mutant (Mut) 3'UTR of DNMT3B mRNA (Shanghai Genechem, Co., Ltd, Shanghai, China) together with $50 \mathrm{nM}$ hsa-miR-26a mimics or negative control (Guangzhou RiboBio) were co-transfected. Transfections were performed using $0.45 \mu 1$ of FuGENE (Promega, Madison, WI, USA). After transfection for $48 \mathrm{~h}$, the luciferase activity was measured by Dual-Glo Luciferase assay system (Promega) with Renilla luciferase activity as control.

Statistical analysis. Data are expressed as means \pm SD. Statistical analysis was performed using the SPSS statistics 20.0. Student's t-test was performed to compare the differences of the two groups. ANOVA was performed for comparison for more than two groups. Correlation between two groups was analyzed using Pearson's correlation coefficient analysis. The level of significance was set at $\mathrm{P}<0.05$. 
A

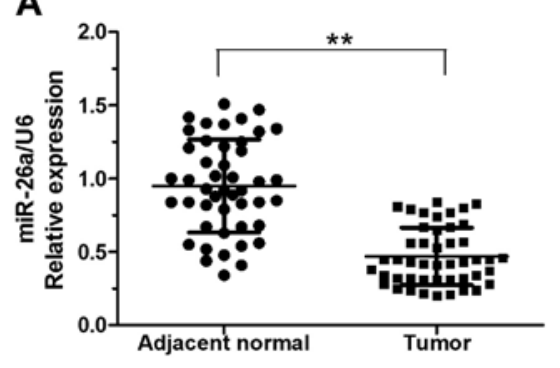

C

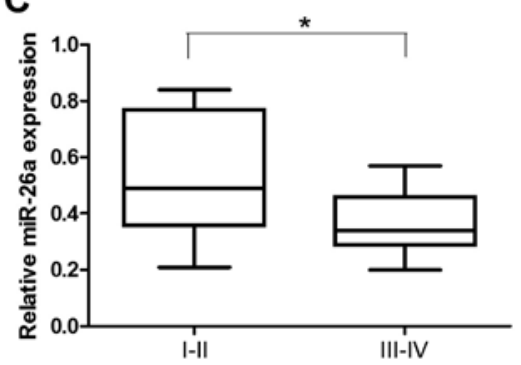

E

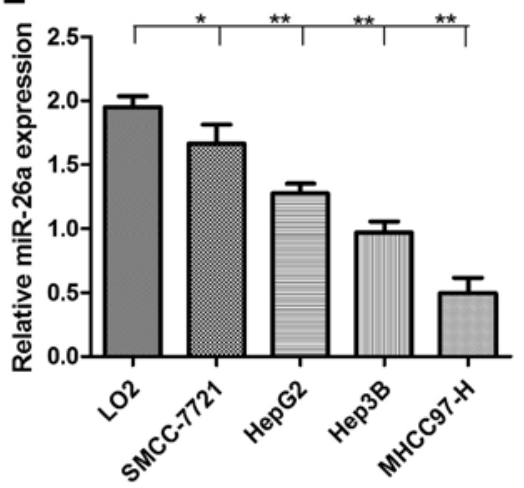

G

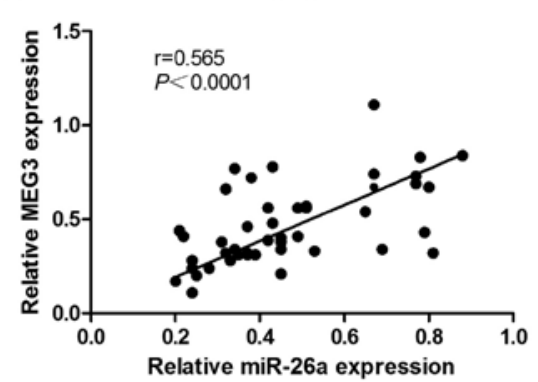

B

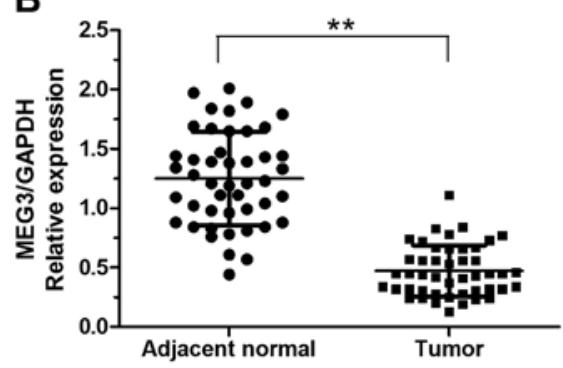

D

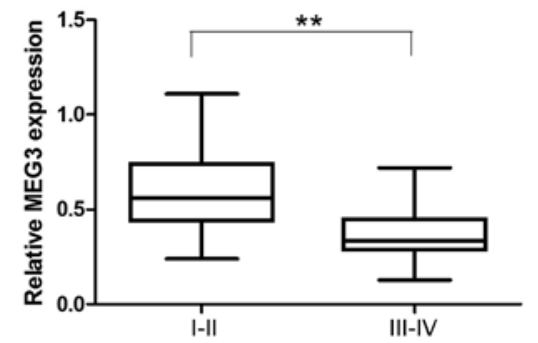

F

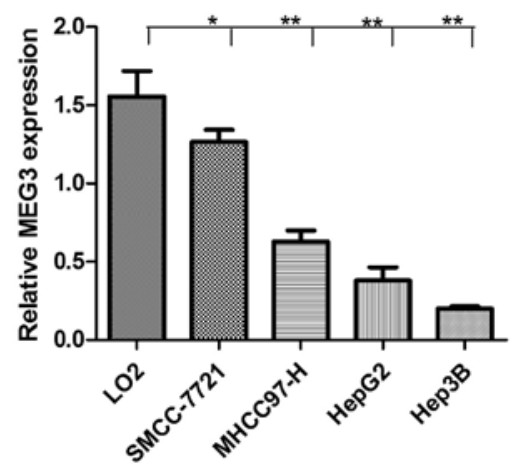

H

Non-malignant

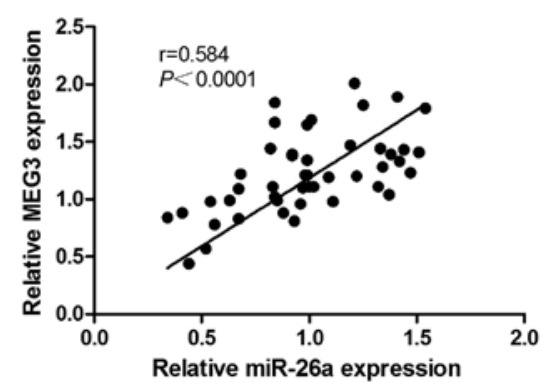

Figure 1. The expression level of miR-26a and MEG3 in HCC tissues and HCC cell lines. (A) miR-26a and (B) MEG3 were downregulated in 46 HCC tissues compared to matched adjacent non-malignant tissues. The expressions of (C) miR-26a and (D) MEG3 in HCC patients with III-IV clinical TNM stages ( $\mathrm{n}=20$ ) were lower than patients with I-II clinical stages $(\mathrm{n}=26)$. The expressions of (E) miR-26a and (F) MEG3 in HCC and LO2 cells were measured using qRT-PCR. Positive correlation between miR-26a and MEG3 expression in $\mathrm{HCC}(\mathrm{G})$ and matched non-malignant tissues $(\mathrm{H})$. Pearson's correlation coefficient analysis parameter $r$ and $\mathrm{P}$-values are shown. ${ }^{*} \mathrm{P}<0.05 .{ }^{* * *} \mathrm{P}<0.01$.

\section{Results}

miR-26a and MEG3 are downregulated in HCC tissues. Expression of miR-26a and MEG3 was analyzed by qRT-PCR in $46 \mathrm{HCC}$ and matched adjacent non-malignant tissues. The relative expression levels of miR-26a and MEG3 were significantly downregulated in HCC tissues compared with matched non-malignant specimens (Fig. 1A and B). Moreover, the expression levels of miR-26a and MEG3 were negatively correlated with the cancer clinical TNM stage, and the advanced TNM stages were found with lower miR-26a and MEG3 expression (Fig. 1C and D). Furthermore, we demonstrated that miR-26a and MEG3 expression levels in the HCC cell lines were lower than normal liver LO2 cells (Fig. 1E and F). In addition, we analyzed the relationship of their expression levels via Pearson's rank correlation coefficient analysis. The result showed that the expression level of miR-26a was positively correlated with MEG3 expression level in both HCC and matched non-malignant tissues (Fig. $1 \mathrm{G}$ and $\mathrm{H}$ ).

Relationship of miR-26a and MEG3 expression with clinicopathological features. The study revealed that the 
Table I. The expression of miR-26a and lncMEG3 and its clinical characteristics in 46 HCC patients.

\begin{tabular}{|c|c|c|c|c|c|}
\hline \multirow[b]{2}{*}{ Clinical factors } & \multirow[b]{2}{*}{ No. } & \multicolumn{2}{|c|}{ miR-26a } & \multicolumn{2}{|c|}{ MEG3 } \\
\hline & & Mean $\pm \mathrm{SD}$ & $\mathrm{P}$-value & Mean $\pm \mathrm{SD}$ & P-value \\
\hline All cases & 46 & & & & \\
\hline Age (years) & & & 0.578 & & 0.207 \\
\hline$<59$ & 20 & $0.489 \pm 0.177$ & & $0.432 \pm 0.190$ & \\
\hline$\geq 59$ & 26 & $0.457 \pm 0.213$ & & $0.504 \pm 0.230$ & \\
\hline Sex & & & 0.855 & & 0.737 \\
\hline Male & 35 & $0.474 \pm 0.191$ & & $0.478 \pm 0.216$ & \\
\hline Female & 11 & $0.462 \pm 0.217$ & & $0.453 \pm 0.215$ & \\
\hline Tumor size (cm) & & & $0.012^{\mathrm{a}}$ & & $0.002^{\mathrm{a}}$ \\
\hline$<5$ & 27 & $0.615 \pm 0.177$ & & $0.566 \pm 0.207$ & \\
\hline$\geq 5$ & 19 & $0.350 \pm 0.112$ & & $0.378 \pm 0.118$ & \\
\hline HBs Ag & & & 0.415 & & 0.341 \\
\hline+ & 34 & $0.456 \pm 0.194$ & & $0.489 \pm 0.231$ & \\
\hline- & 12 & $0.517 \pm 0.206$ & & $0.599 \pm 0.329$ & \\
\hline $\mathrm{AFP}(\mu \mathrm{gl})$ & & & 0.058 & & 0.081 \\
\hline$<20$ & 19 & $0.415 \pm 0.178$ & & $0.412 \pm 0.194$ & \\
\hline$\geq 20$ & 27 & $0.527 \pm 0.207$ & & $0.522 \pm 0.221$ & \\
\hline Histological differentiation & & & $0.009^{\mathrm{a}}$ & & 0.304 \\
\hline Well and moderate & 22 & $0.427 \pm 0.190$ & & $0.505 \pm 0.244$ & \\
\hline Low & 24 & $0.595 \pm 0.165$ & & $0.439 \pm 0.179$ & \\
\hline Clinical (AJCC) stage & & & $0.013^{\mathrm{a}}$ & & $0.004^{\mathrm{a}}$ \\
\hline $\mathrm{I}+\mathrm{II}$ & 26 & $0.514 \pm 0.218$ & & $0.528 \pm 0.204$ & \\
\hline III+IV & 20 & $0.389 \pm 0.112$ & & $0.293 \pm 0.121$ & \\
\hline
\end{tabular}

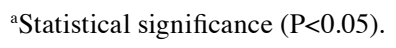

downregulation of miR-26a and MEG3 in HCC were closely associated with the clinicopathological parameters of HCC (Table I). The downregulation of miR-26a had no significant association with patients' age, sex, AFP or HBV infection, but was significantly associated with tumor size, histological differentiation and clinical TNM stage (Table I). As to MEG3 expression, the decreased MEG3 levels were significantly associated with tumor size and TNM stage. However, no statistically significant of its expression was found with the age, sex, AFP, HBV infection or histological differentiation (Table I). These results suggested that downregulation of miR-26a and MEG3 may contribute to the malignant progression of HCC.

miR-26a inhibits HCC cell proliferation, migration and invasion in vitro. To further determine the potential biological effects of miR-26a in HCC, we transfected the miR-26a mimics and compared its performance on HepG2 and MHCC97-H cells. In contrast to negative control group (NC), the ectopic expression of miR-26a suppressed cell proliferation. Transfection of miR-26a mimics for 48, 72 or $96 \mathrm{~h}$ inhibited proliferation of HepG2 cells by $37.5 \%, 38.1 \%$ and $40.1 \%$ (Fig. 2A), MHCC97-H cells by $30.2 \%, 43.2 \%$ and $44.1 \%$, respectively (Fig. 2B). In Matrigel invasion assays (Fig. 2C), upregulation of miR-26a significantly decreased invasion of HepG2 cells (96 vs. 189 cells; $\mathrm{P}<0.01$ ) and MHCC97-H cells (45 vs. 258 cells; $\mathrm{P}<0.01$ ) (Fig. 2E). In migration assays (Fig. 2D), mobility of HepG2 (109 vs. 226 cells; $\mathrm{P}<0.01)$ and MHCC97-H cells ( 119 vs. 288 cells; $\mathrm{P}<0.01)$ (Fig. 2F) were profoundly decreased after transfection of miR-26a mimics.

DNMT3B is a direct target of miR-26a. We used the TargetScan (www.targetscan.org) and found a putative binding site for miR-26a in the 3'-UTR of DNMTB (Fig. 3A). The luciferase reporter assay was conducted to verify the prediction. The wild-type (WT) was transfected with miR-26a mimics and negative control (NC), the relative luciferase activity of WT/miR-26a was significantly decreased compared with WT/NC. However, there was no significant difference observed between Mut/miR-26a and Mut/NC (Fig. 3B). To verified the target relationship between miR-26a and DNMT3B, we measured DNMT3B in HCC cells after transfection of miR-26a mimics. We found that ectopic expression of miR-26a suppressed the protein and mRNA expression levels of DNMT3B in HepG2 and MHCC97-H cells (Fig. 3C and D).

Knockdown of DNMT3B inhibits cell proliferation, migration and invasion. To determine whether downregulation of 

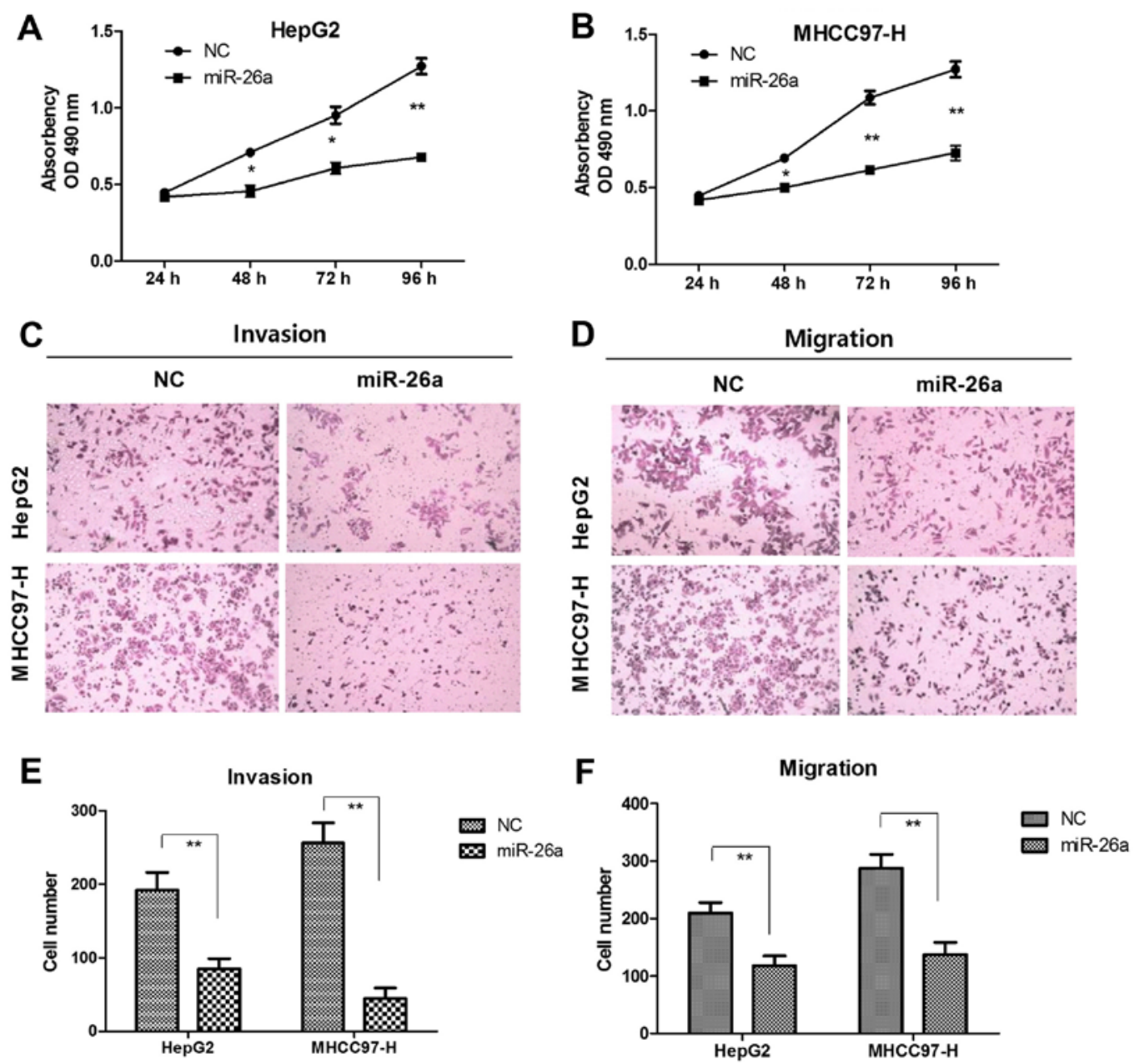

Figure 2. The effects of miR-26a in vitro on proliferation, migration and invasion of HepG2 and MHCC97-H cells. Upregulation of miR-26a significantly inhibited proliferation of (A) HepG2 and (B) MHCC97-H cells. (C) Transwell invasion and (D) migration assays after transfection with miR-26a mimics or negative control $(\mathrm{NC})$ in HCC cells. The number of $(\mathrm{E})$ invaded and $(\mathrm{F})$ migrated cells in the miR-26a-transfected group was significantly decreased compared with negative control. ${ }^{*} \mathrm{P}<0.05 .{ }^{* *} \mathrm{P}<0.01$.
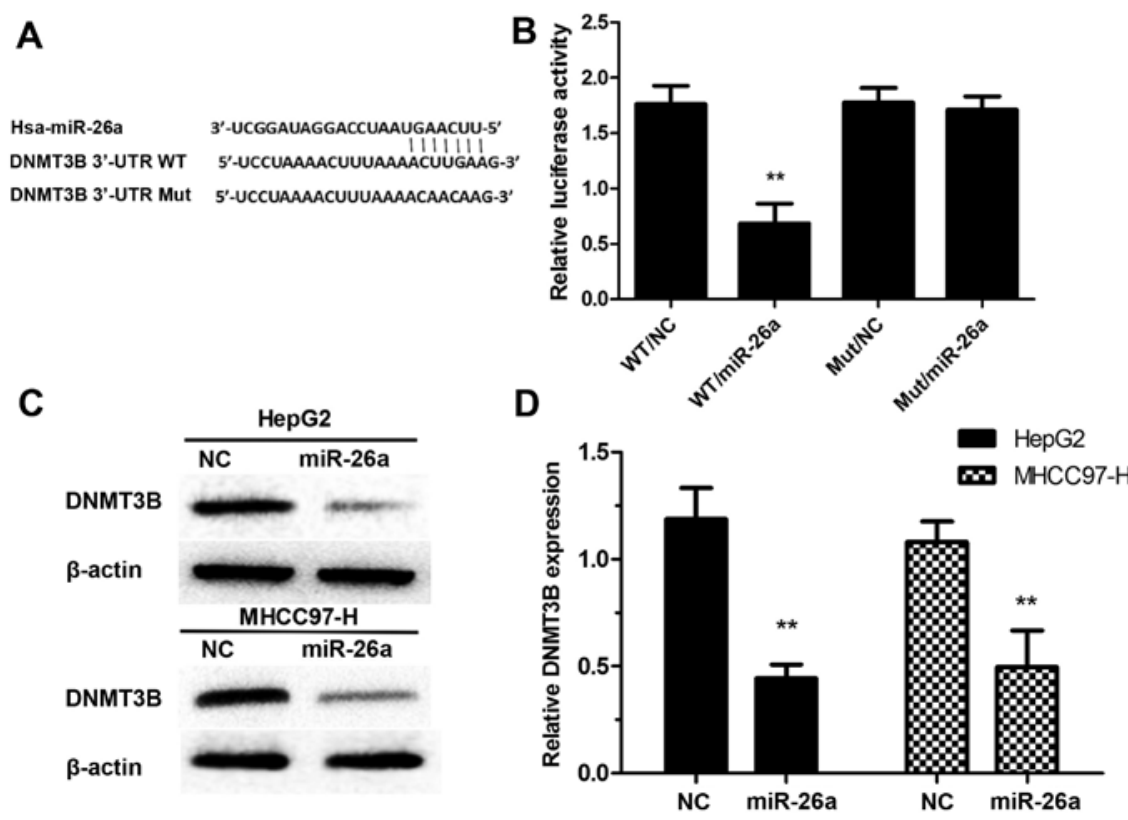

Figure 3. DNMT3B is a direct target gene of miR-26a. (A) The potential binding sequence at the wild-type and mutant DNMT3B mRNA 3'-UTR. (B) Luciferase assay was performed to measure the interaction between DNMT3B 3'-UTR and miR-26a. miR-26a significantly suppressed the luciferase activity that carried WT but not Mut 3'-UTR of DNMT3B. (C) Western blot analysis showed the protein levels of DNMT3B in HepG2 and MHCC97-H cells were downregulated after transfection with miR-26a mimics. (D) Overexpression of miR-26a suppressed the mRNA expression of DNMT3B. ${ }^{* *} \mathrm{P}<0.01$. 
A

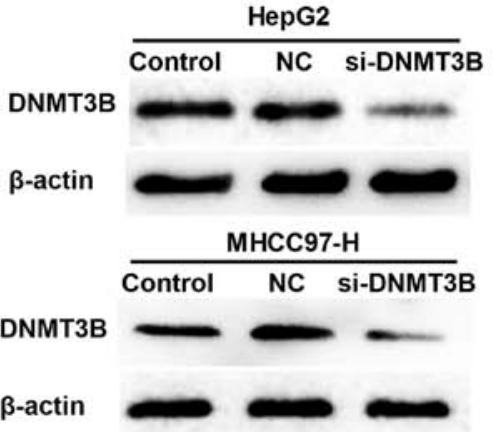

C

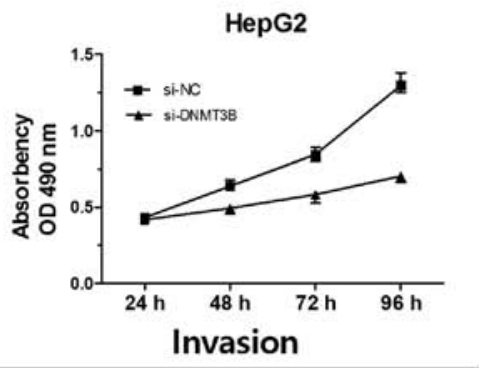

E
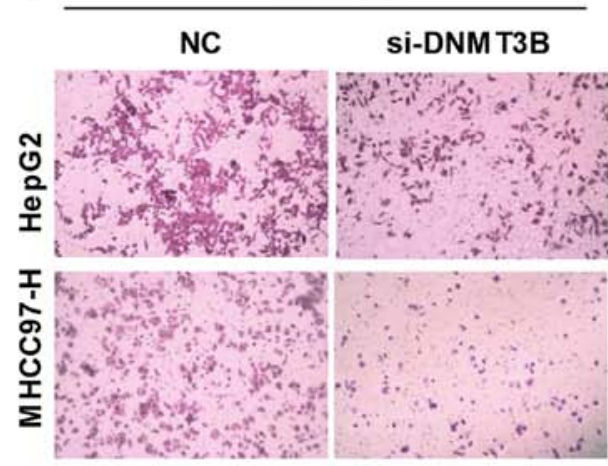

G

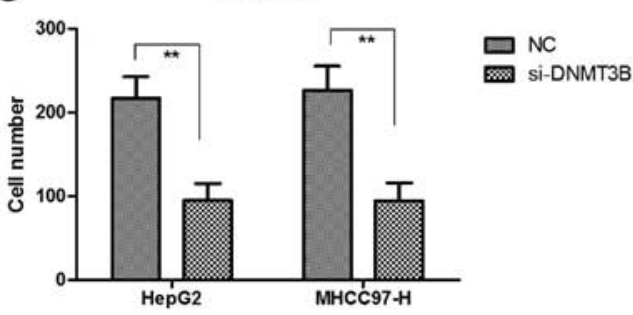

B
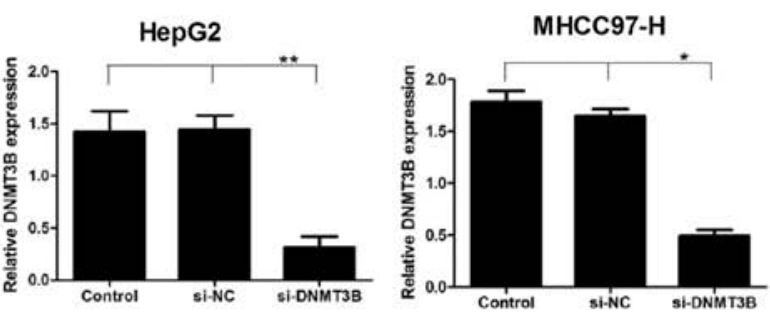

D

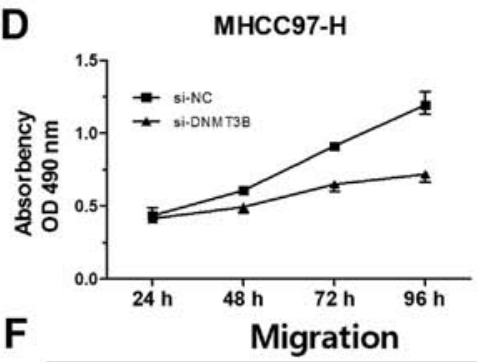

$\mathbf{F}$

NC Si-DNMT3B

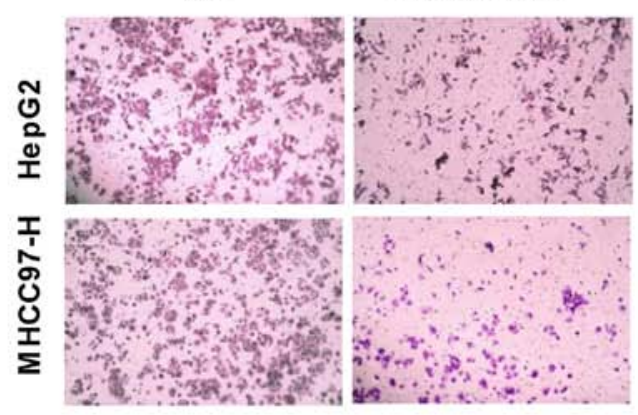

H

Migration

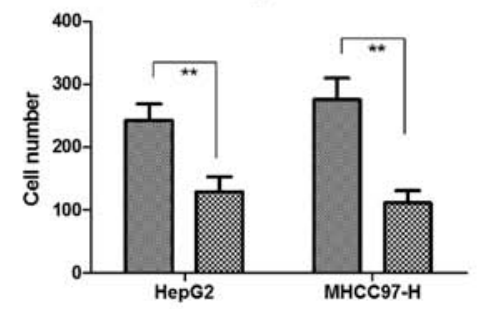

Figure 4. Knockdown of DNMT3B inhibits cell proliferation, migration and invasion in vitro. (A) The protein and (B) mRNA expression of DNMT3B in HepG2 and MHCC97-H cells were downregulated after transfected with si-DNMT3B compared to si-NC. (C and D) MTT assay showed that DNMT3BsiRNA suppressed HepG2 and MHCC97-H cell proliferation. (E and F) Transwell assay showed that DNMT3B-siRNA suppressed HepG2 and MHCC97-H cell invasion and migration. The number of $(\mathrm{G})$ invaded and $(\mathrm{H})$ migrated cells in the si-DNMT3B group was significantly decreased compared with NC group. ${ }^{*} \mathrm{P}<0.05 .{ }^{* *} \mathrm{P}<0.01$.

DNMT3B had similar effect with overexpression of miR-26a, a specific siRNA against the DNMT3B gene transcript was introduced into HCC cells. After transfection with siRNADNMT3B for $48 \mathrm{~h}$, the mRNA and protein expression level of DNMT3B was sharply decreased in HepG2 and MHCC97-H cells (Fig. 4A). Moreover, we found that siRNA-DNMT3B markedly suppressed cell proliferation (Fig. 4B). In addition, the migration and invasion of HCC cells were decreased compared to NC group, respectively (Fig. 4C and D). The effect was consistent with miR-26a overexpression, and indicated that negative regulation of DNMT3B by miR-26a was responsible for miR-26a-induced HCC cell progression at least in part.
miR-26a upregulates MEG3 through inhibition of the DNMT3B expression. In vitro experiment, we found that upregulated miR-26a could increase the expression of MEG3 in HepG2 and MHCC97-H cells (Fig. 5A). Furthermore, knockdown of DNMT3B could also increase MEG3 expression in HepG2 and MHCC97-H cells (Fig. 5B). In combination with our previous findings that miR-26a inhibited the expression of DNMT3B, overexpression of miR-26a may increase the MEG3 expression through reduction of the DNMT3B expression.

MEG3 expression is modulated by DNA methylation. To ascertain the role of aberrant methylation in deregulation of 
A
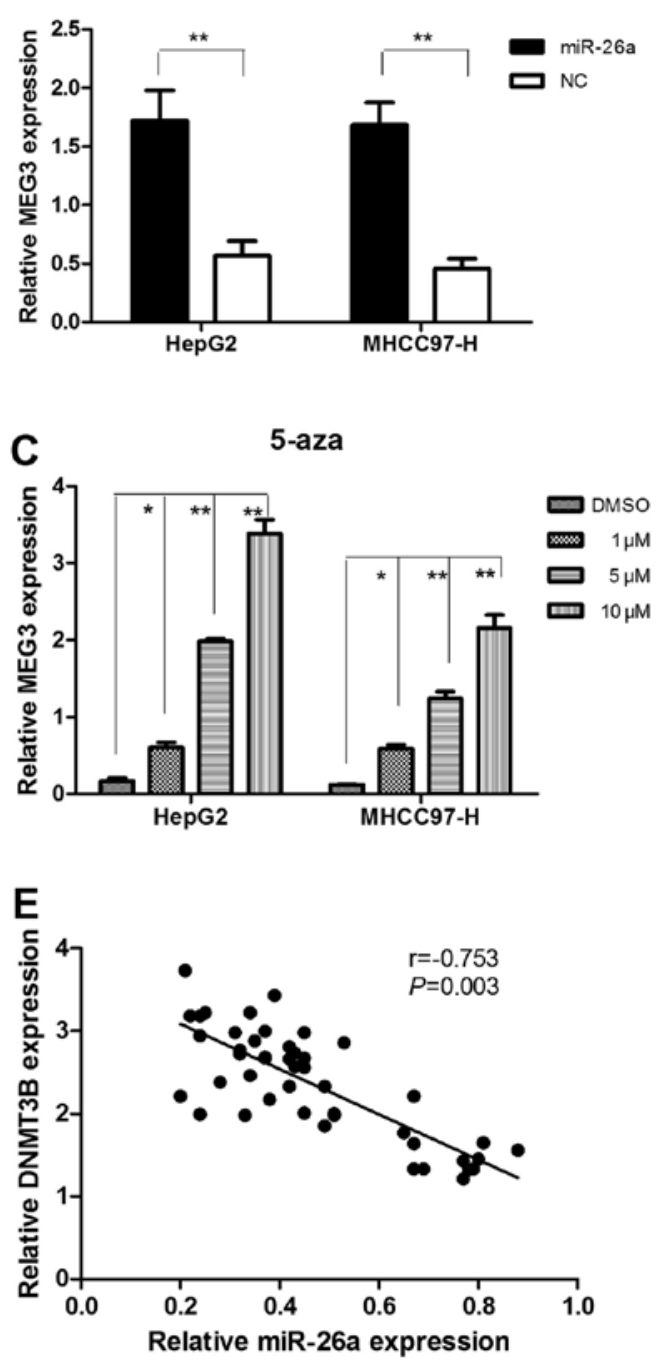

B
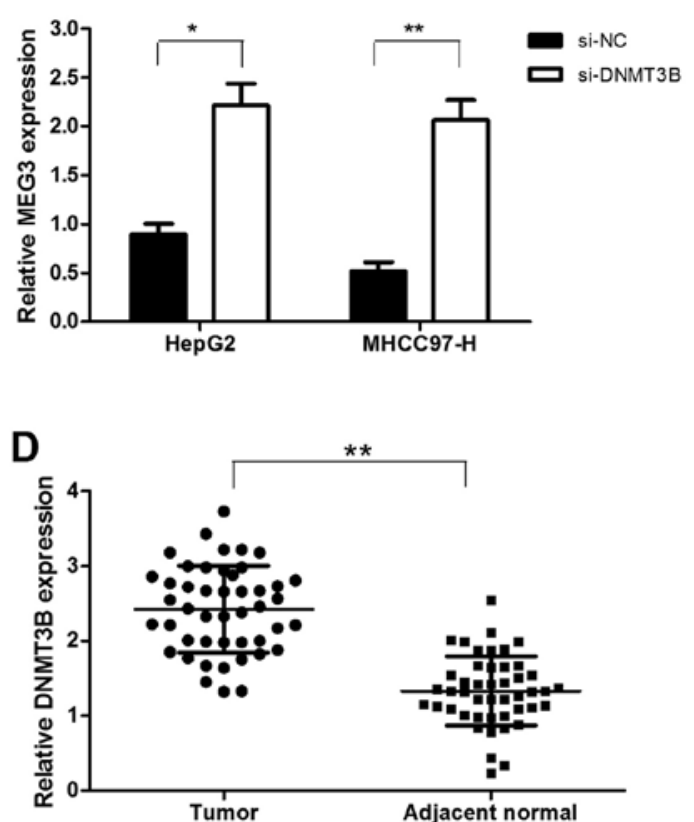

$\mathbf{F}$

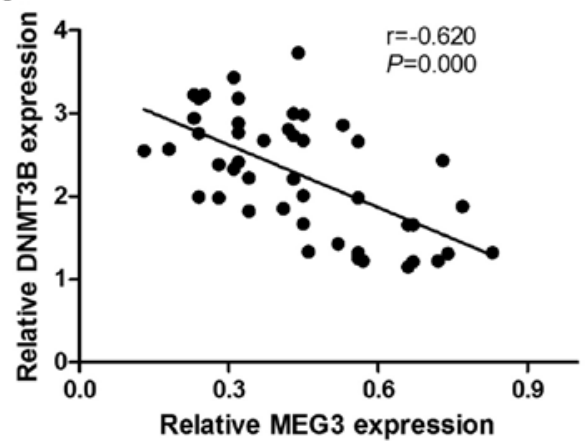

Figure 5. miR-26a increases the MEG3 expression through the inhibition of the DNMT3B expression. (A) Overexpression of miR-26a promoted MEG3 expression in HepG2 and MHCC97-H cells. (B) Inhibition of DNMT3B gene expression downregulated MEG3 expression in HepG2 and MHCC97-H cells. (C) HepG2 and MHCC97-H HCC cells treated with different densities (20, 40, 60 and $80 \mu \mathrm{M})$ of 5-aza-CdR, the expression of MEG3 was upregulated with the increase of the 5-aza-CdR density. (D) The DNMT3B expression was upregulated in HCC compared with matched non-malignant tissues. (E) Pearson's correlation analysis showed negative correlation between miR-26a and DNMT3B expression. (F) Pearson's correlation coefficient analysis showed negative correlation between DNMT3B and MEG3 expression, with $\mathrm{r}$ and $\mathrm{P}$-values as indicated. ${ }^{*} \mathrm{P}<0.05,{ }^{* *} \mathrm{P}<0.01$.

MEG3 in HCC cells, we assessed the effect of a DNA demethylating agent (5-aza-CdR) on MEG3 expression by treating HepG2 and MHCC97-H HCC cells with different densities $(20,40,60$ and $80 \mu \mathrm{M})$. The result showed that MEG3 expression was significantly upregulated with increased density of 5-aza-CdR compared with control (Fig. 5C). It verified again that DNMT3B could imitate the effect of 5-aza-CdR to downregulate the expression of MEG3 in HCC cells.

Upregulation of DNMT3B expression is inversely correlated with miR-26a and MEG3 expression in HCC. To confirm molecular relationship observed in HCC cells, we measured the DNMT3B expression in patient specimens and found that DNMT3B was upregulated in $35(35 / 46,76.1 \%) \mathrm{HCC}$ compared to matched non-malignant tissues (Fig. 5D). Moreover, Pearson's correlation coefficient analysis suggested that DNMT3B expression was inversely correlated with miR-26a and MEG3 expression levels (Fig. 5E and F).

\section{Discussion}

Emerging evidence shows that deregulation of miRNAs play a critical role in tumorigenesis and progression. However, the effect and regulatory mechanisms are often controversial. miR-26a acts as oncogene or tumor suppressor in several human cancers via inhibiting its different target genes. Gao et al (28) found that miR-26a inhibited proliferation and migration of breast cancer through regulating the expression of MCL-1. The study of Deng et al (30) showed that miR-26a suppressed tumor growth and metastasis by targeting FGF9 in gastric cancer. On the contrary, some researchers also found that miR-26a was significantly upregulated in glioblastoma and ovarian cancer $(32,33)$ and promoted lung cancer cell growth through inhibition of PTEN/AKT1 pathway (34). Especially, the biological effect of miR-26a involved in the carcinogenesis of prostate cancer was reported to be in contrast $(35,36)$. Therefore, miR-26a possessed dual effects, 
depending on organ-specific actions and peculiar target genes in different cancer type. In the present study, miR-26a expression was significantly downregulated in HCC compared with matched non-malignant tissues, and low expression of miR-26a was significantly associated with the tumor size, histological differentiation and clinical stage. In vitro, overexpression of miR-26a significantly suppressed the proliferation, migration and invasion of HepG2 and MHCC97-H cells. These results confirmed the inhibitory effect of miR-26a on growth and metastasis in HCC.

miRNAs are suggested to regulate nearly $60 \%$ of proteinencoding genes in mammals post-transcriptionally (37). Recent studies reported that loss of combinations of some miRNAs was associated with hypermethylation defect in breast cancer cell lines, and decreased expression of regulatory miRNAs including miR-26a contributed to DNMT3B overexpression (38). DNMT3B belongs to methyltransferases family and is primarily responsible for methylation of certain genomic regions (39). The overexpression of DNMT3B was observed in some cancer types and contributed to tumor growth and metastasis $(40,41)$. Loss of DNMT3B regulation resulted in complete or partial demethylation of the tumor-suppressive gene MTSS1 promoters and contributed to HCC development (42). Therefore, investigating the contribution of DNMT3B to the process of tumorigenesis is important towards understanding the mechanisms through aberrant DNA methylation in HCC. In the present study, we demonstrated that DNMT3B expression was increased in HCC compared to the adjacent non-malignant tissues, and verified that DNMT3B was a novel target of miR-26. Moreover, knockdown of DNMT3B inhibited cell proliferation, migration and invasion in HepG2 and MHCC97-H cells, which limited the miR-26a-induced tumor suppressive effect. The results suggested that miR-26a regulates proliferation, migration and invasion of HCC cells via inhibition of DNMT3B.

A previous study revealed that MEG3 functions as a tumor-suppressor in HCC, and miR-29a could modulate the expression of MEG3 through the regulation of DNMT3B activity in HCC (31). Other researches reported that DNA methylation could suppress the expression of MEG3 in meningioma and pituitary tumors $(43,44)$. Based on above studies, we assumed that miR-26a could imitate the mechanism to silence the expression of MEG3 in HCC through modulation of DNMT3B. Therefore, we validated our hypothesis in vitro experiments, and the results showed that overexpression of miR-26a increased the expression of MEG3 through reducing expression of its potential target gene DNMT3B in HCC cells. To better confirm the aberrant methylation-dependent mechanism in deregulation of MEG3 in HCC, we introduced the methylation-inhibitor 5-aza-CdR to measure the expression of MEG3. The results showed that MEG3 expression was robustly increased by incubation with 5-aza-CdR in HCC cells, and a concentration-dependent relationship existed. In specimens, the expression of MEG3 was downregulated in HCC compared with non-malignant tissues and showed inverse correlation with either miR-26a or DNMT3B expression in HCC tissues. Based on the above observations, we demonstrated that miR-26a inhibits proliferation and metastasis of HCC by regulating DNMT3B/MEG3 axis.
In summary, we confirmed the tumor suppressor role of miR-26a in HCC, partly at least, via inhibition of DNMT3B/MEG3 axis. The present study complemented the molecular mechanisms of miRNAs in the development of HCC, emphasizing epigenetic modulation, in particular the association of DNA methylation between expression of miRNAs and lncRNAs. A novel molecular regulation axis-miR-26a/DNMT3B/MEG3 may become a promising therapeutic target for HCC treatment. Further studies are needed to investigate the upstream regulatory mechanism underlying miR-26a expression and the downstream signaling pathways of DNMT3B in HCC.

\section{Acknowledgements}

The present study was supported by the National Natural Science Foundation of China (nos. 30771895 and 81502095) and the Key Program of International Cooperation Project of Shaanxi Province, China (no. 2014KW23-04).

\section{References}

1. Bellissimo F, Pinzone MR, Cacopardo B and Nunnari G: Diagnostic and therapeutic management of hepatocellular carcinoma. World J Gastroenterol 21: 12003-12021, 2015.

2. Thomas MB, Jaffe D, Choti MM, Belghiti J, Curley S, Fong Y, Gores G, Kerlan R, Merle P, O'Neil B, et al: Hepatocellular carcinoma: Consensus recommendations of the National Cancer Institute Clinical Trials Planning Meeting. J Clin Oncol 28: 3994-4005, 2010.

3. Crissien AM and Frenette C: Current management of hepatocellular carcinoma. Gastroenterol Hepatol (NY) 10: 3994-4005, 2014.

4. Balogh J, Victor D III, Asham EH, Burroughs SG, Boktour M, Saharia A, Li X, Ghobrial RM and Monsour HP Jr: Hepatocellular carcinoma: A review. J Hepatocell Carcinoma 3: 41-53, 2016.

5. Ferlay J, Shin HR, Bray F, Forman D, Mathers C and Parkin DM: Estimates of worldwide burden of cancer in 2008: GLOBOCAN 2008. Int J Cancer 127: 2893-2917, 2010.

6. Altekruse SF, McGlynn KA and Reichman ME: Hepatocellular carcinoma incidence, mortality, and survival trends in the United States from 1975 to 2005. J Clin Oncol 27: 1485-1491, 2009.

7. Zhao ZC, Zheng SS, Wan YL, Jia CK and Xie HY: The molecular mechanism underlying angiogenesis in hepatocellular carcinoma: The imbalance activation of signaling pathways. Hepatobiliary Pancreat Dis Int 2: 529-536, 2003.

8. Kawaji H, Severin J, Lizio M, Forrest AR, van Nimwegen E, Rehli M, Schroder K, Irvine K, Suzuki H, Carninci P, et al: Update of the FANTOM web resource: From mammalian transcriptional landscape to its dynamic regulation. Nucleic Acids Res 39 (Database): D856-D860, 2011.

9. Amorim M, Salta S, Henrique R and Jerónimo C: Decoding the usefulness of non-coding RNAs as breast cancer markers. J Transl Med 14: 265, 2016.

10. Beermann J, Piccoli MT, Viereck J and Thum T: Non-coding RNAs in development and disease: Background, mechanisms, and therapeutic approaches. Physiol Rev 96: 1297-1325, 2016.

11. Croce CM: Causes and consequences of microRNA dysregulation in cancer. Nat Rev Genet 10: 704-714, 2009.

12. Arora A, Singh S, Bhatt AN, Pandey S, Sandhir R and Dwarakanath BS: Interplay between metabolism and oncogenic process: Role of microRNAs. Transl Oncogenomics 7: 11-27, 2015.

13. Bartel DP: MicroRNAs: Genomics, biogenesis, mechanism, and function. Cell 116: 281-297, 2004.

14. Yu X, Li Z, Yu J, Chan MT and Wu WK: MicroRNAs predict and modulate responses to chemotherapy in colorectal cancer. Cell Prolif 48: 503-510, 2015.

15. Jansson MD and Lund AH: MicroRNA and cancer. Mol Oncol 6: 590-610, 2012.

16. Wen Y, Han J, Chen J, Dong J, Xia Y, Liu J, Jiang Y, Dai J, Lu J, Jin G, et al: Plasma miRNAs as early biomarkers for detecting hepatocellular carcinoma. Int J Cancer 137: 1679-1690, 2015. 
17. Hu S, Tao R, Wang S, Wang C, Zhao X, Zhao H, Li L, Zhu S, He Y, Jiang X, et al: MicroRNA-21 promotes cell proliferation in human hepatocellular carcinoma partly by targeting HEPN1. Tumour Biol 36: 5467-5472, 2015.

18. Zheng F, Liao YJ, Cai MY, Liu YH, Liu TH, Chen SP, Bian XW, Guan XY, Lin MC, Zeng YX, et al: The putative tumour suppressor microRNA-124 modulates hepatocellular carcinoma cell aggressiveness by repressing ROCK 2 and EZH2. Gut 61: 278-289, 2012

19. Zhang JP, Zeng C, Xu L, Gong J, Fang JH and Zhuang SM: MicroRNA-148a suppresses the epithelial-mesenchymal transition and metastasis of hepatoma cells by targeting Met/Snail signaling. Oncogene 33: 4069-4076, 2014.

20. Lai YY, Shen F, Cai WS, Chen JW, Feng JH, Cao J, Xiao HQ, Zhu GH and $\mathrm{Xu}$ B: MiR-384 regulated IRS1 expression and suppressed cell proliferation of human hepatocellular carcinoma. Tumour Biol 37: 14165-14171, 2016.

21. Xue D, Zhou C, Lu H, Xu R, Xu X and He X: LncRNA GAS5 inhibits proliferation and progression of prostate cancer by targeting miR-103 through AKT/mTOR signaling pathway. Tumour Biol: Oct 14, 2016 (Epub Epub ahead of print).

22. Yim GW, Kim HJ, Kim LK, Kim SW, Kim S, Nam EJ and Kim YT: Long non-coding RNA HOXA11 antisense promotes cell proliferation and invasion and predicts patient prognosis in serous ovarian cancer. Cancer Res Treat: Oct 11, 2016 (Epub Epub ahead of print).

23. Wang J, Ye C, Xiong H, Shen Y, Lu Y, Zhou J and Wang L: Dysregulation of long non-coding RNA in breast cancer: an overview of mechanism and clinical implication. Oncotarget 8 : 5508-5522, 2016

24. Liu YR, Tang RX, Huang WT, Ren FH, He RQ, Yang LH, Luo DZ, Dang YW and Chen G: Long noncoding RNAs in hepatocellular carcinoma: Novel insights into their mechanism. World J Hepatol 7: 2781-2791, 2015.

25. Xia T, Liao Q, Jiang X, Shao Y, Xiao B, Xi Y and Guo J: Long noncoding RNA associated-competing endogenous RNAs in gastric cancer. Sci Rep 4: 6088, 2014.

26. Hu L, Ye H, Huang G, Luo F, Liu Y, Liu Y, Yang X, Shen J, Liu Q and Zhang J: Long noncoding RNA GAS5 suppresses the migration and invasion of hepatocellular carcinoma cells via miR-21. Tumour Biol 37: 2691-2702, 2016.

27. Deng J, He M, Chen L, Chen C, Zheng J and Cai Z: The loss of miR-26a-mediated post-transcriptional regulation of cyclin E2 in pancreatic cancer cell proliferation and decreased patient survival. PLoS One 8: e76450, 2013.

28. Gao J, Li L, Wu M, Liu M, Xie X, Guo J, Tang H and Xie X: MiR-26a inhibits proliferation and migration of breast cancer through repression of MCL-1. PLoS One 8: e65138, 2013.

29. Lin Y, Chen H, Hu Z, Mao Y, Xu X, Zhu Y, Xu X, Wu J, Li S, Mao Q, et al: miR-26a inhibits proliferation and motility in bladder cancer by targeting HMGA1. FEBS Lett 587: 2467-2473, 2013.

30. Deng M, Tang HL, Lu XH, Liu MY, Lu XM, Gu YX, Liu JF and He ZM: miR-26a suppresses tumor growth and metastasis by targeting FGF9 in gastric cancer. PLoS One 8: e72662, 2013.
31. Braconi C, Kogure T, Valeri N, Huang N, Nuovo G, Costinean S, Negrini M, Miotto E, Croce CM and Patel T: microRNA-29 can regulate expression of the long non-coding RNA gene MEG3 in hepatocellular cancer. Oncogene 30: 4750-4756, 2011.

32. Guo P, Lan J, Ge J, Nie Q, Guo L, Qiu Y and Mao Q: MiR-26a enhances the radiosensitivity of glioblastoma multiforme cells through targeting of ataxia-telangiectasia mutated. Exp Cell Res 320: 200-208, 2014.

33. Shen W, Song M, Liu J, Qiu G, Li T, Hu Y and Liu H: MiR-26a promotes ovarian cancer proliferation and tumorigenesis. PLoS One 9: e86871, 2014

34. Liu B, Wu X, Liu B, Wang C, Liu Y, Zhou Q and Xu K: MiR-26a enhances metastasis potential of lung cancer cells via AKT pathway by targeting PTEN. Biochim Biophys Acta 1822: 1692-1704, 2012

35. Zhao S, Ye X, Xiao L, Lian X, Feng Y, Li F and Li L: MiR-26a inhibits prostate cancer progression by repression of Wnt5a. Tumour Biol 35: 9725-9733, 2014.

36. Guo K, Zheng S, Xu Y, Xu A, Chen B and Wen Y: Loss of miR-26a-5p promotes proliferation, migration, and invasion in prostate cancer through negatively regulating SERBP1. Tumour Biol 37: 12843-12854, 2016.

37. Melo SA and Esteller M: Dysregulation of microRNAs in cancer: Playing with fire. FEBS Lett 585: 2087-2099, 2011.

38. Sandhu R, Rivenbark AG and Coleman WB: Loss of post-transcriptional regulation of DNMT3b by microRNAs: A possible molecular mechanism for the hypermethylation defect observed in a subset of breast cancer cell lines. Int J Oncol 41: 721-732, 2012.

39. Nishida N, Nishimura T, Nakai T, Chishina H, Arizumi T, Takita M, Kitai S, Yada N, Hagiwara S, Inoue T, et al: Genomewide profiling of DNA methylation and tumor progression in human hepatocellular carcinoma. Dig Dis 32: 658-663, 2014

40. Chen WC, Chen MF and Lin PY: Significance of DNMT3b in oral cancer. PLoS One 9: e89956, 2014.

41. Liu Z, Wang L, Wang LE, Sturgis EM and Wei Q: Polymorphisms of the DNMT3B gene and risk of squamous cell carcinoma of the head and neck: A case-control study. Cancer Lett 268: 158-165, 2008.

42. Fan H, Chen L, Zhang F, Quan Y, Su X, Qiu X, Zhao Z, Kong KL, Dong S, Song Y, et al: MTSS1, a novel target of DNA methyltransferase 3B, functions as a tumor suppressor in hepatocellular carcinoma. Oncogene 31: 2298-2308, 2012.

43. Zhao J, Dahle D, Zhou Y, Zhang X and Klibanski A: Hypermethylation of the promoter region is associated with the loss of MEG3 gene expression in human pituitary tumors. J Clin Endocrinol Metab 90: 2179-2186, 2005.

44. Zhang X, Gejman R, Mahta A, Zhong Y, Rice KA, Zhou Y, Cheunsuchon P, Louis DN and Klibanski A: Maternally expressed gene 3 , an imprinted noncoding RNA gene, is associated with meningioma pathogenesis and progression. Cancer Res 70: 2350-2358, 2010. 